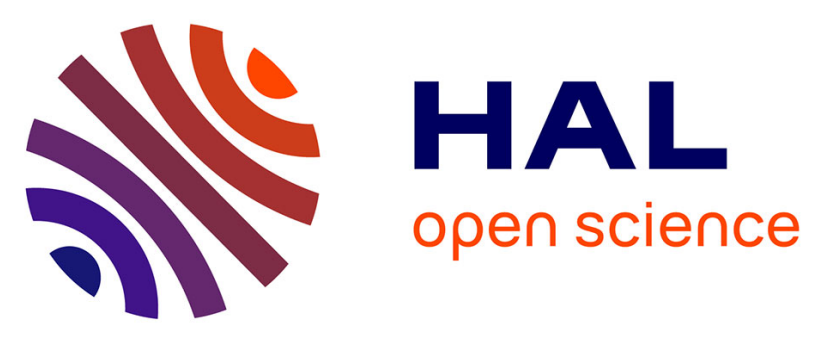

\title{
Des chamois dans les Vosges
}

Louis Badré, François Tassel

\section{To cite this version:}

Louis Badré, François Tassel. Des chamois dans les Vosges. Revue forestière française, 1956, 4, pp.305-308. 10.4267/2042/27194 . hal-03383959

\section{HAL Id: hal-03383959 \\ https://hal.science/hal-03383959}

Submitted on 18 Oct 2021

HAL is a multi-disciplinary open access archive for the deposit and dissemination of scientific research documents, whether they are published or not. The documents may come from teaching and research institutions in France or abroad, or from public or private research centers.
L'archive ouverte pluridisciplinaire HAL, est destinée au dépôt et à la diffusion de documents scientifiques de niveau recherche, publiés ou non, émanant des établissements d'enseignement et de recherche français ou étrangers, des laboratoires publics ou privés. 


\section{DES CHAMOIS DANS LES VOSGES}

M. le Conservateur BADRÉ qui a reçu les chamois capturés et M. l'Ingénieur Principal TASsel qui a organisé leur reprise en Allemagne ont bien voulu adresser à la Revue forestière le récit de, cet essai d'acclimatation et nous les en remercions.

Le C. de R.

Comme l'a précisé lẹ Docteur Couturier (I), l'espèce Rupicapra Rupicapra est une espèce pleistocène à phylum jeune - son aire naturelle actuelle s'étend de l'Espagne à l'Asie-Mineure; plus étendue en longitude qu'en latitude, elle comprend les chaînes de montagne suivantes de l'Ouest à l'Est et du Nord au Sud: Monts Cantabriques, Pyrénées, Alpes, une partie des Carpathes et de la Transylvanie, Péninsule Balkanique, chaine Pontique et Taurus en AsieMineure, Caucase.

A l'intérieur de cette aire, l'espèce revêt des formes géographiques ne présentant entre elles que des différences morphologiques légères; par exemple en France le chamois et l'isard; on distingue parfois des sujets dits " de forêt " plus trapus et des sujets dits " de rocher ) ou de " glacier " plus longilignes.

Deux essais d'acclimatation de cette espèce ont bien réussi :

Le premier dans une île du Sud de la Nouvelle Zélande, qui se trouve sur un parallèle à peu près symétrique, par rapport à l'équateur, de celui de Burgos (Espagne).

Le deuxième, en Forêt-Noire, au Feldberg, que l'on peut, semblet-il, considérer plutôt comme une réacclimatation.

En effet, jusqu'au $\mathrm{XIV}^{\ominus}$ siècle (I), la présence de chamois était à peu près permanente dans ce massif atteignant $\mathrm{I} 500 \mathrm{~m}$ et où les pelouses de type alpin sont assez abondantes au voisinage des crêtes, où le vent chasse la forêt.

Poursuivi de plus en plus par l'homme, le chamois disparait alors jusqu'à nos jours, on ne note plus, de temps à autre que le passage d'animaux isolés venant de Suisse et de l'Allgau et montant vers le Feldberg.

C'est ainsi qu'en 'I932, une chèvre élit domicile dans la vallée du Zastler, reliant Kirchzarten, au Sud de Fribourg-en-Brisgau, aux pentes Nord du Feldberg.

(I) Dr Couturier. Le chamois.

(2) Oskar Küchler. Landesforstmeister. Wild und Hund, I956, n ${ }^{\circ} 22$. 
En I935, le Landesjägermeister. Hug décide de tenter un essai d'acclimatation et en avril de la même année, on lâche dans cette vallée un bouc, capturé en Yougoslavie ainsi qu'un bouc et trois chèvres venant de Gmünden (Autriche). Ces 5 animaux se dispersent rapidement; un bouc est braconné, la trace des autres est perdue.

Deux nouveaux lâchers ont lieu, l'un en I938 (3 boucs et 5 chèvres), l'autre en I939 ( 3 boucs et 4 chèvres), tous en provenance de Gmünden.

Cette fois, les animaux restent groupés dans le Zostlerthal, s'y multiplient et se répandent aux alentours.

Sur l'initiative de M. Meyer, Conservateur des Eaux et Forêts et chef du service forestier français pour le Pays de Bade, le Général Commandant en chef prend une ordonnance classant en réserve intégrale de chasse tout le territoire du Feldberg.

En I955, l'effectif total est estimé à 600 têtes; le tir du chamois est alors autorisé pour la première fois par les adjudicataires, selon un plan établi par le service forestier.

Désirant témoigner sa reconnaissance à la France pour la mesure de sauvegarde prise en I945, le gouvernement allemand propose au Conseil Supérieur de la Chasse de lui remettre gracieusement un lot de chamois à reprendre au Feldberg.

L'offre est acceptée et il est lécidé que les anintaux qui auront été capturés seront relâchés sur le versant alsacien des Hautes-Vosges, entre le Grand-Ballon et le Markstein où le biotope est sensiblement comparable à celui offert par le Feldberg.

Le 7 janvier, il est procédé à la reprise dans le Zastlerthal, sur le versant du Rinken, à une altitude comprise entre 900 et I $200 \mathrm{~m}$ où se tiennent généralement 30 à 40 chamois.

600 mètres de panneaux servant aux reprises de cerfs à Chanbord ont été prêtés aux services allemands par le Conseil Supérieur de la Chasse.

Il est décidé de faire deux battues; à cette fin $500 \mathrm{~m}$ de panneaux sont placés le long d'une ligne de plus grande pente au milieu du versant; ils constituent le petit côté du premier rectangle à traquer ; les deux grands côtés, parallèles aux courbes de niveau, sont ceinturés de banderoles sur environ I kilomètre; Ioo $\mathrm{m}$ de panneaux sont également placés en retour et dans le haut du versant - IO2 bûcherons dirigés par 8 agents forestiers exécutent la manœuvre; 22 capteurs se répartissent le long des 600 mètres de panneaux et se placent so $\mathrm{m}$ en avant de ceux-ci - 40 hommes soutiennent les $2000 \mathrm{~m}$ de banderoles et les agitent lorsqu'un animal paraitra vouloir les forcer; les traqueurs sont au nombre de 40.

L'état-major forestier dispose en outre de 6 voitures radios de la police, permettant des liaisons d'un bout à l'autre et de haut en bas clu versant à parcourir; d'un vétérinaire et d'un médecin. Le 
matériel a été amené à pied d'œuvre, malgré une couche de neige de Io à $20 \mathrm{~cm}$, un peu verglacée par places, grâce à deux unimogs.

En ce qui concerne la capture proprement dite et par conparaison avec celle des cerfs et chevreuils par le même procédé, il semble que celle du chamois soit plutôt aisée; les animaux ont paru avoir tendance à descendre la pente plutôt qu'à la remonter; une fois capturés, ils ont manifesté un calme imperturbable, reprenant très rapidement une respiration normale. Le cerf et le chevreuil donnent, manifestement, d'autres signes d'énervement, de crainte ou d'impatience.

Le total cles captures s'élève à I 3 (soit environ $2 / 3$ des animaux rencontrés) - 2 jeunes chèvres sont relâchées; il est conservé et mis en caisse:

-2 boucs de 2 ans,

- 5 chèvres de 2 à 7 ans,

- 2 chevreaux mâles,

- 2 chevreaux femelles.

La harde est assez bien constituée en ce qui concerne la répartition des sexes, mais on doit regretter l'absence de mâles adultes.

Le transport des animaux s'effectue sans encombre par camions; le Rhin est franchi à la tombée de la nuit, au bureau de douanes de Chalampé et vers 22 heures, le convoi arrive en forêt communale de Ramspach, dans la vallée de Saint-Amarin (700 m d'altitude).

Après avoir été immatriculés et auscultés, les I I animaux sont relâchés les uns après les autres; dans l'obscurité, ils ne vont pas très loin et ne disparaissent qu'une fois regroupés.

L'emplacement a été choisi pour sa tranquillité - la forêt de Ranspach a le triste privilège d'être la plus mal desservie en chemins de tout le Haut-Rhin -, pour le sérieux de l'adjudicataire de la chasșe -, pour son relief accidenté -, elle culmine au Markstein -, et pour sa proximité de la région la plus rocheuse et la plus escarpée des Vosges, au Sud du Hohneck où l'on pense que ces chamois chercheront leur gite définitif.

En fait, 8 jours après leur arrivée dans les Vosges, ils ont été aperçus, toujours groupés, à $I_{5} \mathrm{~km}$ environ au. Nord de leur point de départ et à mi-chemin de cette région escarpée du Herrenberg et de la Worms.

Que penser de l'avenir de ce troupeau?

Au point de vue chasse, un arrêté préfectoral a été pris le ro janvier I956 en interdisant le tir sur tout,le territoire du Haut-Rhin.

Quant à l'habitat, le chamois peut trouver au-dessus de I $200 \mathrm{~m}$ dans les Vosges des régions aussi escarpées sinon plus que dans la Forêt Noire. Malheureusement, les Vosges profondément découpées par de nombreuses vallées, sont plus habitées et très fréquentées par les touristes et les zones tranquilles y sont rares. 
La végétation du massif du Hohneck, quoique légèrement différente de celle du Feldberg, en raison des influences atlantiques plus nettes, et des différences de sol, granitique ici et gneissique à l'Est du Rhin, présente quelques plantes alpines (anemona alpina, viola lutea, androsace carnea, etc...) qui permettent de supposer que le chamois trouvera dans son nouvel habitat des conditions favorables.

Et puisqu'il a été constaté en Forêt Noire qu'il se nourrit presque exclusivement de lichens ou de graminées, et qu'il ne cause aucun dégât aux forêts au milieu desquelles il vit, il reste au Forestier d'Alsace à lui souhaiter de s'adapter à sa nouvelle station, de s'y multiplier comme en Bade et d'attester ainsi par sa présence des deux côtés de la vallée du Rhin, la communauté d'idéal qui unit les Forestiers de tous les pays.

L. B. et F. T.

\section{Vous lirez dans}

\section{La Revue du Bois}

de mars I956:

Influence de la vitesse sur le travail des dents de scie, par R. ANTOINE.

Le bouchon de liège, par A. Dugelay.

L'écorçage chimique des bois, par J. RIsI.

Le rabotage, par E. LEDINOT. 